\title{
Nonvalvular myocardial involvement in metastatic carcinoid disease
}

\author{
Gershan Davis, Kevin Birbeck, David Roberts, Naseer Naqvi
}

\section{Summary}

A patient with the unusual post mortem finding of myocardial metastatic carcinoid tumour without classical valvular or endocardial carcinoid disease is described. This rare occurrence may represent an aggressive type of carcinoid tumour, with metastatic disease occurring before the development of classical fibrous valvular and endocardial pathology.

Keywords: carcinoid heart disease, myocardium, metastatic disease

Carcinoid heart disease occurs in approximately $50 \%$ of patients with the carcinoid syndrome and results in impaired overall survival. The tricuspid and pulmonary valves are primarily involved in this condition, although other recognised lesions include leftsided valvular disease, and tumour deposition in the atria and large vessels. We report an unusual case of metastatic carcinoid tumour within the left ventricular free wall without 'classical' valvular involvement.

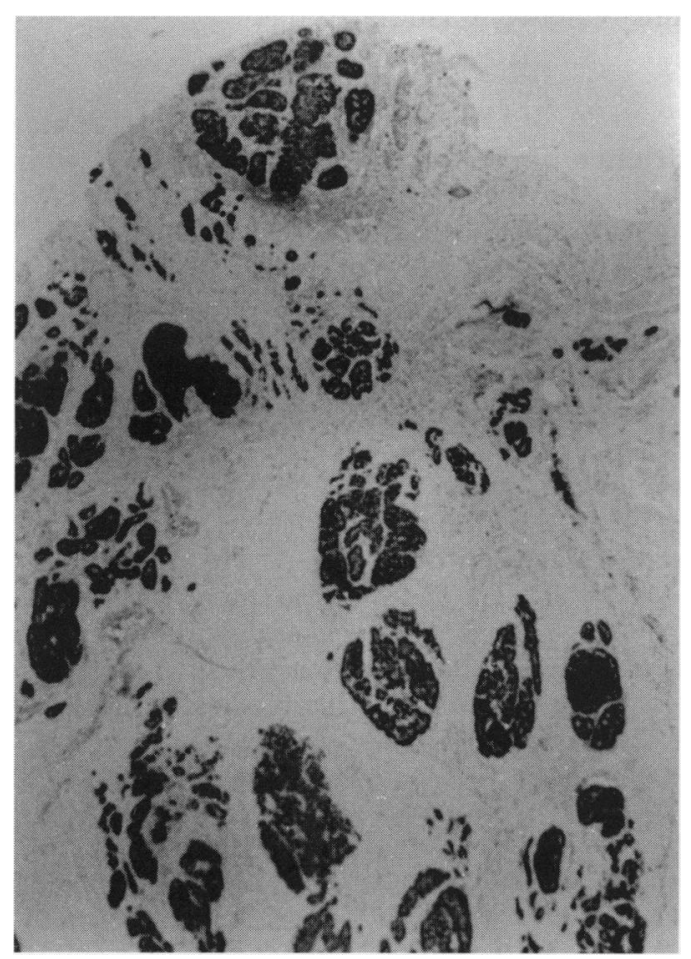

Figure 1 Photomicrograph of carcinoid tumour in the terminal ileum. (The epithelium has sloughed off the luminal surface.) Chromogranin stain

\section{Case report}

A previously fit 75 -year-old man presented with an acute confusional state. Two months previously he had developed a progressive symmetrical polyarthropathy affecting the hands and feet and was treated with nonsteroidal agents and hydroxychloroquine. While on the above medication he developed a generalised maculopapular rash and his medication was discontinued. He became progressively lethargic, febrile and confused. He later complained of headaches and abdominal pain and on examination was flushed with bronchospasm and tender hepatomegaly.

Resting electrocardiogram showed uncontrolled atrial fibrillation which subsequently reverted spontaneously to sinus rhythm. Chest $\mathrm{X}$-ray and abdominal ultrasound were normal. Computed tomography (CT) scan of the brain did not show any space-occupying lesions.

Eight days later he became severely jaundiced and was found to have an autoimmune haemolytic anaemia with the presence of cold agglutinins. He died soon afterwards despite resuscitative therapy. At post mortem an invasive

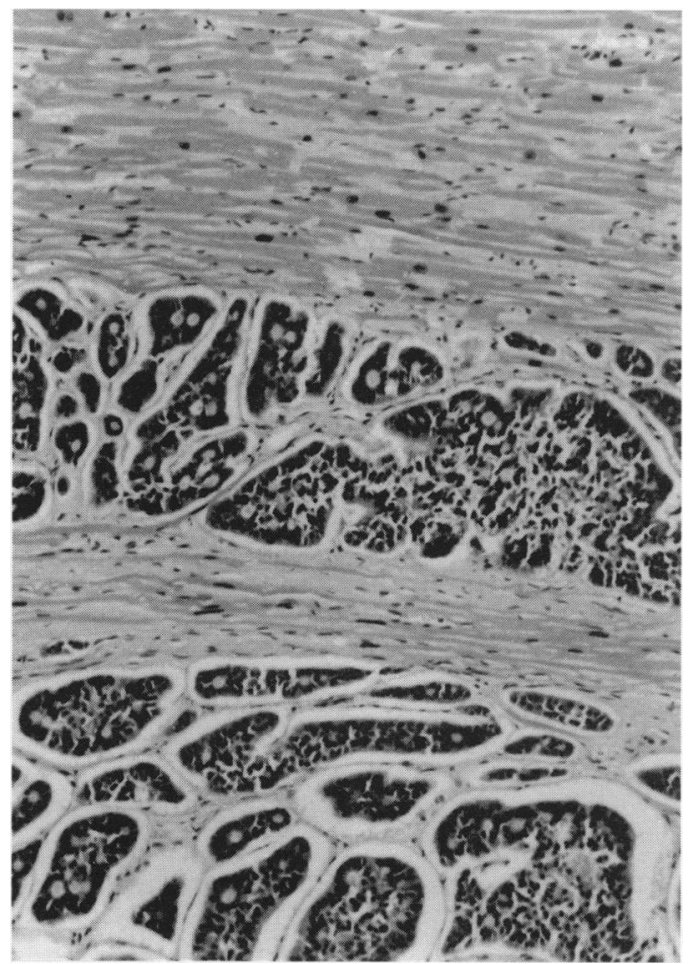

Figure 2 Photomicrograph of the left ventricular myocardial free wall demonstrating metastatic carcinoid tumour. Chromogranin stain 
carcinoid tumour of the terminal ileum was found (figure 1) with widespread metastases including deposits in the lymph nodes of the porta hepatis, brain, liver, right atrial myocardium and left ventricular myocardium (figure 2). There were no valvular or endocardial lesions.

\section{Discussion}

Myocardial metastatic carcinoid tumour is a recently described finding. Pellikka et al claim to have first reported its occurrence in their review of 74 patients with carcinoid heart disease. They found it in three cases (4\%), but in two of these there was also classical carcinoid disease involving the right-sided heart valves. There has been one other case. of carcinoid deposition within the left ventricular free wall. ${ }^{2}$ As in our case, this was confirmed on histological examination, also

1 Pellikka PA, Tajik AJ, Khandheria BK, et al. Carcinoid heart disease, clinical and echocardiographic spectrum in 74 patients. Circulation 1993; 87: 1188-96.

2 Schiller VL, Fishbein MC, Siegel RJ. Unusual cardiac involvement in carcinoid syndrome. Am Heart $₹ 1986 ; 112$ :

$1322-3$.
Types of carcinoid heart disease

- endocardial disease: right-sided valvular disease, commonly tricuspid regurgitation and pulmonary stenosis; left-sided valvular disease (aortic and/or mitral); restrictive cardiomyopathy

- metastatic myocardial disease: atrium or ventricle

- pericardial disease: pericardial effusion

- vascular involvement: eg, coronary sinus, aorta

with an absence of valvular or endocardial carcinoid plaques. It is likely that these three cases represent an aggressive variant of carcinoid tumour, as the classical endocardial lesions appear to represent chronic exposure to elevated levels of serotonin and other vasoactive substances. ${ }^{3}$

3 Trell E, Rausing A, Ripa J, Torp A, Waldenstrom J. Carcinoid heart disease. Clinicopathologic findings and follow-up in 11 cases. Am $\mathcal{f}$ Med 1973; 54: 43.

\title{
A patient with hepatitis $B$, antimicrosomal antibodies, and autoimmune hypothyroidism
}

\author{
Wilson R Catapani, Orsine Valente, Claudia Aguiar
}

\begin{abstract}
Summary
A 37-year-old man with chronic hepatitis $B$ was diagnosed as having antimicrosomal antibodies and subclinical hypothyroidism. The association of autoimmune manifestations with hepatitis $B$ has been less frequently reported than with hepatitis $C$ virus. It is discussed whether this patient illustrates a case of spontaneous development of antimicrosomal antibodies, only casually associated with the presence of hepatitis $B$ virus, or there is a real causative relationship between both conditions.
\end{abstract}

Keywords: hepatitis B virus, antimicrosomal antibodies, hypothyroidism

Hepatitis $B$ virus $(\mathrm{HBV})$ and hepatitis $C$ virus (HCV) are among the main hepatotropic human viruses. Besides being aetiologic agents of chronic liver damage, it has been speculated that they can act as triggers of autoimmune diseases. There are reports of the association of HCV with cryoglobulinaemia and thyroid autoantibodies $^{1,2}$ and the development of an autoimmune chronic active hepatitis after an acute infection with $\mathrm{HBV}^{3}$ However, the coexistence of HBV and thyroid disease has rarely been reported. We describe the case of a young man with chronic hepatitis $\mathbf{B}$ who presented subclinical hypothyroidism and thyroid antimicrosomal antibodies.

\section{Case report}

A 37-year-old man was found to be HBsAG positive after a screening test for blood donation. $\mathrm{He}$ was totally asymptomatic when he presented for medical advice. His physical examination was normal. No risk factor related to $\mathrm{HBV}$ infection was identifiable. HBV serological markers were as follows: HBsAG positive, anti-HBsAG negative, anti-HBcAG positive, HBeAG-negative, anti-HBeAG positive. A needle liver biopsy showed chronic lobular hepatitis, with negative immunoperoxidase staining for HBsAG and HBcAG in the hepatic parenchyma. He had normal alkaline phosphatase, $\gamma$-glutamyl transferase and coagulation tests; aspartate transaminase and alanine transaminase were slightly elevated to the upper normal limit. A test for anti-HCV 